See Article page 1261.

\section{Commentary: Endovascular repair to the rescue!}

\author{
Syed T. Hussain, MD, and John A. Goncalves, MD
}

Intercostal and visceral patch aneurysm after previous open surgical repair (OSR) of thoracoabdominal aortic aneurysm (TAAA) is a challenging problem. ${ }^{1,2}$ The use of endovascular and hybrid techniques as an alternative to OSR for this indication is increasing, but the challenges remain formidable irrespective of the treatment modality. ${ }^{3}$ However, the literature is limited to small series or case reports.

In this issue of the Journal, Tenorio and colleagues ${ }^{4}$ present the results of a multicenter international study of 29 patients treated for intercostal and visceral aortic patch aneurysms after previous open TAAA repair with fenestratedbranched endovascular aortic repair (F-BEVAR). ${ }^{4}$ They used either patient-specific devices or off-the-shelf t-Branch stent grafts or physician-modified endografts. A total of 103 target arteries were incorporated by 54 fenestrations and 49 directional branches, with a median of 4 target arteries per patient. The authors achieved $100 \%$ technical success, defined as deployment of the aortic stent graft and all intended side branch components. There was no operative mortality, and only 5 patients $(17 \%)$ had a major adverse event. No patient had paraplegia or required new-onset dialysis; however, 9 patients $(31 \%)$ required secondary intervention, performed with a percutaneous endovascular approach in 8 of these cases $(89 \%)$. Freedom from reintervention at 2 years was $61 \%$.

This study represents the largest experience of F-BEVAR for recurrent TAAA, and the authors provide excellent relevant technical details and discuss important anatomic and procedural challenges. These include graft design, reduced

\footnotetext{
From the Donald and Barbara Zucker School of Medicine at Hofstra/Northwell, Hempstead, NY; and Department of Cardiovascular and Thoracic Surgery, Northwell Health/South Shore University Hospital, Bay Shore, NY.

Disclosures: The authors reported no conflicts of interest.

The Journal policy requires editors and reviewers to disclose conflicts of interest and to decline handling or reviewing manuscripts for which they may have a conflict of interest. The editors and reviewers of this article have no conflicts of interest.

Received for publication April 19, 2021; revisions received April 19, 2021; accepted for publication April 20, 2021; available ahead of print April 27, 2021.

Address for reprints: Syed T. Hussain, MD, Thoracic and Cardiovascular Surgery, South Shore University Hospital, 301 E Main St, Bay Shore, NY 11706 (E-mail: hussaintarique@hotmail.com).

J Thorac Cardiovasc Surg 2023;165:1273-4

$0022-5223 / \$ 36.00$

Copyright (c) 2021 by The American Association for Thoracic Surgery

https://doi.org/10.1016/j.jtcvs.2021.04.057
}

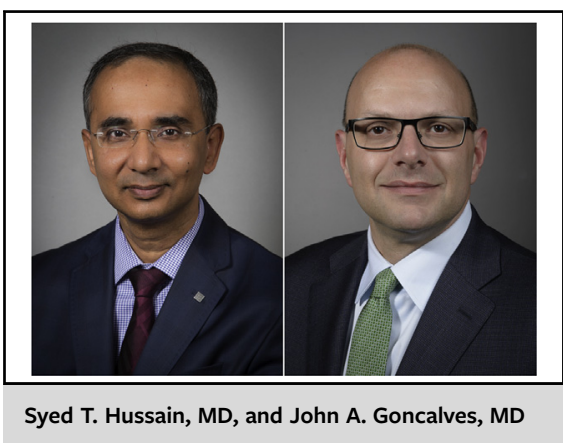

CENTRAL MESSAGE

Fenestrated branched endovas-

cular repair of intercostal or

visceral aortic patch aneurysm is

a feasible option in highly expe-

rienced centers, albeit with sig-

nificant need for secondary

intervention.

landing zone compliance, limited luminal room for guidewire and catheter manipulation, distortion of visceral vessel anatomy induced by patch aneurysmal degeneration or use of bypass reconstructions, and limitation of device manipulation in the presence of a surgical graft. Prevention of spinal cord ischemia is critical, and an understanding of the technical considerations and graft design is key to minimizing this dreaded complication.

Despite the small number of patients, the lack of comparative group who underwent OSR, and the high rate of secondary interventions, the authors report outcomes that compare favorably to reoperative OSR outcomes. Coselli and colleagues ${ }^{2}$ did not detect any noteworthy differences in early outcomes between reoperative TAAA repair (726 patients) and non-reoperative TAAA repair (2653 patients); however, they reported an operative mortality of $11 \%$ and other significant morbidities for visceral and intercostal patch aneurysms treated by OSR. The high rate of secondary interventions after F-BEVAR likely reflects the anatomic complexity and technical challenges associated with visceral and aortic patch aneurysms, which should decline with continued improvement in stent-graft design and implantation techniques.

The authors have answered the most relevant question: can intercostal and visceral patch aneurysm after previous OSR of TAAA be treated with endovascular repair? Despite 
the high secondary reintervention rate, their results compare favorably with those of open reoperative OSR, supporting the feasibility of F-BEVAR for this indication. It must be emphasized that these results represent the experience of highly experienced centers and cannot be generalized. The long-term durability of F-BEVAR remains to be determined, especially in younger patients and patients with connective tissue disorders. Future studies comparing OSR and F-BEVAR, although difficult given the relatively small number of patients, are needed to provide a more definitive answer.

\section{References}

1. Afifi RO, Sandhu HK, Trott AE, Nguyen TC, Miller CC, Estrera AL, et al. Redo thoracoabdominal aortic aneurysm repair: a single-center experience over 25 years. Ann Thorac Surg. 2017;103:1421-8.

2. Coselli JS, Rosu C, Amarasekara HS, Green SY, Zhang Q, Price MD, et al. Reoperative surgery on the thoracoabdominal aorta. J Thorac Cardiovasc Surg. 2018; 155:474-85.e1.

3. Bertoglio L, Mascia D, Cambiaghi T, Kahlberg A, Tshomba Y, Gomez JC, et al. Management of visceral aortic patch aneurysms after thoracoabdominal repair with open, hybrid, or endovascular approach. J Vasc Surg. 2018;67:1360-71.

4. Tenorio ER, Oderich GS, Schanzer A, Beck AW, Gargiulo M, Farber MA, et al Endovascular repair of intercostal and visceral aortic patch aneurysms following open thoracoabdominal aortic aneurysm repair. J Thorac Cardiovasc Surg. 2023;165:1261-71.e5. 